\title{
$\mathrm{V}$ \\ UROLOGICAL CONDITIONS IN RELATION TO VENEREAL DISEASE*
}

\author{
By J. SWIFT JOLY, F.R.C.S.
}

Madam President, Ladies and Gentlemen,-There is no doubt it is for the benefit of the patient that there are specialists, and it is for the benefit of the profession at large that there should be specialists; but I sometimes wonder whether it is good for the specialist himselfwhether he does not become narrow and have his views restricted to one small portion of the subject of general medicine. I sometimes also wonder whether he does not tend to work in a water-tight compartment, and more or less ignore those who are working in neighbouring fields.

It was with very great pleasure I accepted your kind invitation to me to come here, because it gives me the opportunity of saying something about the border-line between venereal disease and urology, and I expect I shall learn as much from the ensuing discussion as you will from my address.

The first point I want to make concerns diagnosis. We all make mistakes, some of them excusable, some not. The urologist may ignore or pass over a chronic prostatitis and be very much surprised if the patient develops gonorrhœal urethritis three days after the examination. A venereal disease specialist may consider that the chronic pyuria from which the patient is suffering, is due to the urethral focus, and not to some disease in the upper urinary tract. A few years ago I operated on a young man for stone in the bladder. He had a calculus the size of a walnut, and had been treated for chronic gonorrhœa for a period of three and a half years. After removal of the stone the pus cleared from the urine, and there was no sign of urethral infection. Another patient had a chronic pyuria with staphylococcus albus. He had been under treatment for a considerable time at one of the large clinics, and had been admitted into hospital to

* An Address delivered before the Medical Society for the Study of Venereal Diseases, January $25^{\text {th, }} 1935$. 


\section{UROLOGICAL CONDITIONS}

hasten his cure. After remaining as an-in-patient for a month he was discharged to have a rest from all treatment. The day after he got home he passed no urine. He was brought into an L.C.C. hospital, and I saw him thirty-six hours after the commencement of anuria. I cut down on the right side and found a stone in the upper end of the right ureter, which I removed. On X-ray examination another stone was found in a corresponding position on the other side ; that also I removed. I kept him under observation for a long time, because I was anxious to find out whether he had two totally different conditions, or whether the pyuria was due to the calculi. But repeated examinations failed to reveal any sign of urethritis.

It should be possible to avoid such mistakes, and it generally is possible if the last half-ounce of urine in the patient's bladder is examined separately from the remainder of the specimen. If that last half-ounce is free from pus, the source of the pyuria must be below the bladder. If there is pus in that specimen, the source of the trouble is in the bladder itself, or in the kidney. That addition to the ordinary test is a very useful one ; it has saved me from error on several occasions.

Talking of tests, there is a point I have often wondered about: I can never make out why the two-glass test has such a tremendous vogue. It seems to me to be useless in the way it is generally employed at present. It was introduced by Sir Henry Thompson fifty years ago with the idea of distinguishing between the pyuria arising from the bladder, and that in the kidneys. The patient was asked to pass urine into two glasses. The first of them was discarded. Any pus in that was assumed to be a contamination from the urethra, and examination for his urinary condition was made from the second glass. As a test in gonorrhœa that test does not seem to me to be at all satisfactory. The test I employ is one I found was habitually used at St. Peter's Hospital when I went there as house surgeon in 1907. It is performed as follows. The anterior urethra is washed out with a clear solution, and any threads, flakes, or pus in it are judged to come from this portion of the canal. The patient is then asked to pass a sample of urine, but not to empty his bladder completely. This carries away the pus or débris from the posterior urethra. The prostate and/or 


\section{BRITISH JOURNAL OF VENEREAL DISEASES}

the seminal vesicles are then massaged, and their contents expressed into the urethra. The patient then passes another sample of urine which contains the secretion of these glands. Finally the last half-ounce or so of urine in his bladder is passed into another glass, and it shows the state of the upper urinary tract. This test is accurate enough for all practical purposes, and does not take long to carry out. I only mention it as the two-glass test is still often recommended, especially in foreign text-books.

By means of this test it is easy to watch the onset of posterior urethritis in cases of acute gonorrhœa. Long before the patient has any symptoms of infection in the posterior urethra, pus and débris can be collected from this part of the canal, and one can watch the gradual increase in these inflammatory products. It is also interesting to note the proportion of cases in which a posterior urethritis develops. On looking over my old case sheets, I found that it developed in all the cases which were first seen two or more days after the appearance of the discharge, in 75 per cent. of cases seen during the second day, and in a little less than 50 per cent. of cases seen during the first day. This is additional evidence, if additional evidence is really needed, of the importance of early treatment in acute gonorrhœa.

In the treatment of gonorrhøa the surgeon has two aims. The first is to destroy the gonococci as quickly as possible, and the second is to prevent the invasion of secondary organisms. A man with living gonococci in his urethra is a menace to society, but it is not always recognised that one with a secondary infection may be a menace to himself. It is largely in connection with these secondary organisms that I wish to speak to-night.

Looking over my old notes, I noticed that the invasion of secondary organisms was practically absent in cases where I carried out all the treatment myself, but it became a serious factor when the treatment was either entirely neglected, or carried out by the patient. Every urologist knows that infection always follows selfcatheterisation, and in many instances proves fatal; but one may be inclined to overlook the fact that the patient who injects or irrigates his urethra is exceedingly liable to infect the canal. The average patient will listen attentively while the methods of self-irrigation or self-injection are explained, but very few of them will at the same time 


\section{UROLOGICAL CONDITIONS}

take in the principles of asepsis. If the necessity of cleanliness has been impressed on him, he soon becomes careless, and as his first lapse is rarely followed by dire results, he continues in his careless habits. I feel certain that in clinics where the treatment of acute gonorrhœa is carried out entirely by the surgeon or by a well-trained orderly, the percentage of secondary infections will be much smaller than in those where the treatment is carried out by the patient himself.

There are various types of secondary infections. In some cases a great number of organisms are to be seen on the film together with a comparatively small number of pus cells. This type is, in my opinion, comparatively harmless, and clears up rapidly under suitable treatment. In other cases there is a chronic pyuria with only a small number of organisms visible in a stained film. In these cases it is often necessary to cultivate the organism in order to identify it. This type is, I believe, far more dangerous, and is much more prone to give rise to longcontinued urinary sepsis. In such cases the pyogenic cocci are usually the infecting organisms. The staphylococcus albus is the most common, but all forms of staphylococci may be found. Streptococci are seldom present alone, but a mixed infection of "streps" and "staphs" is extremely resistant to treatment.

A chronic coccal infection of this type is often dismissed as being harmless and of no consequence either to the patient or to those with whom he may have connection. This is a mistaken view. The infection may persist for years. It tends to spread widely in the urinary tract, and is often the origin of calculous disease or pyonephrosis occurring in later life, and lastly there is an increasing amount of evidence that it may be spread through the sexual act. This last point deserves more attention than it has hitherto received. The following case may serve as an example. Some years ago I was consulted by a man who had been married six weeks previously, and whose wife had developed salpingitis. He stated that eight years before he developed gonorrhœa, for which he was treated for two years. He was then pronounced cured, but he had a residual infection due to the staphylococcus albus ever since. Before his marriage he had consulted several urologists in America and France, all of whom said that the infection was non- 


\section{BRITISH JOURNAL OF VENEREAL DISEASES}

specific and harmless. On the strength of this he married. His wife was under the care of a gynæcologist who failed to find any evidence of a gonococcal infection, and who asked me to see the husband in order to ascertain if the husband could still be infectious. I found a very chronic prostatitis, and was always able to cultivate the staphylococcus albus from the expressed secretion. There was no evidence of a gonococcal infection. This case is suggestive, but it is not conclusive. It shows, however, that there is a risk in allowing a man with a chronic non-specific urethritis to marry, and that the surgeon should be exceedingly cautious in giving his consent. On the other hand, there are cases where marriage appears to be harmless for the female and beneficial for the male.

There is another reason why I lay so much stress on this type of chronic non-specific infection. I believe it to be the origin of most of the unfortunate sequelæ of gonorrhœa. The gonococcus does not, in my opinion, tend to persist indefinitely in the urethra. Cabot announced the comforting doctrine that it usually died out in about two years. I cannot agree with this statement, but I have only twice seen it persist for more than seven years. An organism which tends to become extinct in a comparatively short time cannot be held responsible for a stricture developing ten, fifteen, or even twenty years after an attack of gonorrhœa. Again, in cases of chronic stricture one very rarely finds the gonococcus, except when there is a history of recent re-infection. I, therefore, believe that in most cases, a chronic stricture is not the result of a pure gonococcal infection, but of a non-specific urethritis following gonorrhœa. The following case is, however, a direct contradiction of this statement. About three years ago a young man, about twenty-five, consulted me for stricture. He said that he had been infected when a baby by a Chinese nurse, and had never had connection in his life. The first part of the story was afterwards confirmed by his father. During the previous year symptoms of obstruction occurred, and a stricture was diagnosed by his local doctor. I found a very tight stricture at the level of the suspensory ligament, for which he has been continuously under treatment. When he first consulted me a mixture of gonococci, "staphs," and "streps" were found in his urine. After about a year the gonococcus disappeared, but the pyogenic cocci 


\section{UROLOGICAL CONDITIONS}

are still present. This patient had no treatment during the interval of about twenty-two years that elapsed between subsidence of his original infection, and the onset of obstructive symptoms, yet the gonococcus must have persisted in his urethra for all those years. In addition it was present in sufficient numbers to be readily recognisable in every smear that was taken. The other organisms were only identified by culture.

I think that one is justified in describing two types of urethral stricture. The first occurs early, usually within a year of the gonorrhœal infection. The stricture is usually linear, and only involves a very short portion of the urethra. The calibre is as a rule fairly large, so that there are no obstructive symptoms, and as there may be little or no difficulty in passing a sound, they can only be diagnosed by means of the urethroscope. In fact they are usually found on a routine examination. Lastly, the mucous membrane is healthy down to the face of the stricture. If the infection has died out, or been cured, they do not tend to contract. I have urethroscoped many of these patients at regular intervals for periods up to ten years from the time of the original gonorrhœa, without finding any tendency for the stricture to contract, provided the urine remained sterile. As a rule, however, I took the precaution to dilate the urethra fully before discharging the patient. In all the cases of this type I have seen, the secondary infection was absent or insignificant.

The second type comprises the chronic stricture cases which crowd out all urological out-patient departments. The patient usually presents himself because he has difficulty in micturition, and this does not occur till several years after his attack of gonorrhœa. On urethroscopy there is usually a stenosis at the level of the suspensory ligament, which is narrow enough to hold up an ordinary urethroscope tube. The whole of the perineal urethra is more or less strictured, and under air distension, ring after ring of contraction, each narrower than that in front of it, can be seen extending down to a tight stricture in the bulb. In addition, the mucous membrane of the whole urethra is profoundly altered. The blood-vessels, usually so apparent in the normal urethra, are quite invisible. The light yellow colour of the normal urethral mucosa is changed to a dirty grey 


\section{BRITISH JOURNAL OF VENEREAL DISEASES}

tint, and there is evidence of long-continued infection in all the follicles of Morgagni. In these cases it is rare to find the gonococcus, but there always is a severe nonspecific infection, and all sorts of organisms can be obtained from the urine or from a smear. In addition, there is a large amount of pus in the urine. In these cases the stricture always tends to recontract, and the patient requires life-long treatment.

I have described these two conditions as different types of stricture. It would be more correct to designate them as different degrees. Every bad stricture must pass at some time through a stage where the stenosis resembled that described as the first type. The sole difference between the two is that in the second type the infection persisted, while in the first it cleared up. The infection is always non-specific. This brings us back to my original thesis, that every care should be taken to prevent a secondary infection by means of careful treatment in the initial stages of an acute gonorrhœa. It may not be possible to stamp out the disease, but if treatment can be devised by which the onset of a secondary infection can be absolutely prevented, then gonorrhœa will be shorn of its devastating sequelæ. The whole future of a gonorrhœa patient often depends on the treatment he receives during the first two or three weeks of his illness, and to my mind the great advantage of the new Venereal Clinics is that the patients receive careful treatment during this period.

Turning once more to the question of stricture, we have in the past been inclined to lay too much stress on the mechanical obstruction, and too little on the infection. The latter gradually spreads until the whole of the urinary tract becomes involved. A man develops a stricture, the infection spreads to the periurethral tissues and gives rise to perineal abscesses and urinary fistulæ. It also spreads upwards involving the prostate and/or the seminal vesicles, the bladder, and finally reaches the kidneys. When both kidneys are infected, the patient becomes a chronic invalid, and his life is materially shortened.

The following is the history of one of these cases. The patient is now aged thirty-five. He contracted gonorrhœa when he was twenty. His treatment was intermittent and incomplete, and he left the clinic before he was 


\section{UROLOGICAL CONDITIONS}

discharged as cured. About five years later he began to have difficulty in micturition, and two or three years after this he developed an abscess in his perineum, which was allowed to burst of itself. This was followed by a second abscess, and later by a third, and so on. I first saw him about five years ago, that is, about ten years after his original gonorrhœea. $\mathrm{He}$ had a very dense stricture, and a great mass of inflammatory fibrous tissue in his perineum, which was riddled with sinuses discharging pus and urine. I dissected out this mass, and did an internal urethrotomy. He did very well for a time, and then disappeared. He returned recently with a very foul cystitis, and severe pain in the right loin. The urethra took a No. I2 English fairly easily, and a radiogram showed a large stone in the pelvis of the right kidney, which was functionless. As the urine from the left kidney was sterile, I removed the right. It was a pyonephrosis without any excretory value. During his convalescence he developed a left-sided pyelitis, with a temperature rising up to 105. This was relieved by tying in a catheter. This patient is still a young man, his only kidney is now infected, and the remainder of his urinary tract is also involved. I do not think that he can live for more than a few years. This is the final result of a single attack of gonorrhœea. Fortunately these cases are rare when compared with the enormous number of acute gonorrhœas seen every year.

Even if the infection spreads no higher than the prostate it can still be a serious menace. A chronic prostatitis is frequently followed by an overgrowth of fibrous tissue in the gland. This in turn involves the internal sphincter, and transforms it into a rigid inelastic ring. Patients with this condition develop serious obstruction, with all its harmful effects on the kidneys. This form of prostatic obstruction-the obstruction due to the " small, fibrous prostate" -is usually the direct result of a post-gonorrhœal non-specific infection, with or without true stricture formation. If untreated, it is just as fatal as the obstruction caused by the enlarged adenomatous gland. It usually occurs in patients about fifty, that is, distinctly below the " prostatic age." Up to the present, treatment has been difficult, as one has to remove not only a small, fibrous, adherent gland, but the neck of the bladder as well. Recently good results have 


\section{BRITISH JOURNAL OF VENEREAL DISEASES}

been obtained by per-urethral resection, but in many cases an open operation is to be preferred.

About two years ago, one of these patients was admitted under me into St. Peter's Hospital. He had marked obstruction from a small fibrous gland, in which a radiogram showed a few small calculi. His blood urea was then well above the $200 \mathrm{mg}$. per Ioo c.c. A suprapubic cystotomy was performed, and the patient was discharged with a permanent drainage apparatus. $\mathrm{He}$ returned a couple of weeks ago, and after two years' drainage the blood urea was still Io8. As his general condition was good I decided to operate, and removed the prostate together with the internal sphincter. He is making satisfactory progress, but is still in hospital.

True prostatic calculi are so often associated with stricture and chronic prostatitis that I think most cases of this condition must be considered to be sequels of gonorrhœe. If the calculi do not give rise to symptoms I do not advise any treatment. If there is residual urine, I usually treat the stricture first. If the patient is still unable to empty his bladder completely, I remove the gland. This relieves the obstruction, but, of course, the patient must keep his stricture well dilated.

There is another question I wish to raise. It is the relationship between true urinary calculi and gonorrhœa. At St. Peter's Hospital about 43 per cent. of all renal calculi are infected at the time of the operation. It is not possible to say if the infection is primary, or if the stones formed in sterile urine, which afterwards became infected. Grossmann, from von Lichtenberg's clinic, found that 42 per cent. were infected at the time of operation. He definitely states that the source of infection was gonorrhœal, and that there was a residual infection of the internal genital organs from which the kidneys became contaminated. The commonest organism he found was the staphylococcus albus, and he also found similar cocci in material expressed from the prostate and seminal vesicles. Hellström has shown that in some cases of this type the infection is primary. This he demonstrated by dissolving the calcareous portion of the stone, and then cutting sections of the remaining colloid framework. He found clumps of organisms in the centre of the stone, which probably formed the nucleus of the calculous formation. In a few instances he was able to 


\section{UROLOGICAL CONDITIONS}

cultivate the organisms, and usually proved them to be the staphylococcus albus, in other cases he was unable to go further than to say that they were Gram-positive cocci. He concludes that the staphylococcus albus is a specific stone-former, and that it precipitates the lime salts and has the power of building them into concretions. At St. Peter's Hospital we also find the staphylococcus albus is very frequently associated with stone formation, but I do not think there is sufficient evidence to say that it has any specific action. It tends to render the urine alkaline. This alone is sufficient to cause a precipitation of calcium phosphate, which is the principal constituent of these stones. The crystals become deposited on the surface of the organisms in accordance with the general principle of adsorption. On this theory, the rôle of the cocci is secondary, and they only create conditions favourable for stone formation. Still the fact remains, that if there was no infection the stones would probably not form. There is also some evidence that the infection originated as a post-gonorrhœal urethritis.

The last point I wish to mention is the question of sterility after gonorrhœa. It is well known that sterility frequently follows double epididymitis, owing to blockage of the vas. Much can be done by anastomosing the vas to the upper pole of the epididymis, and good results following this operation have been reported from America. I have also had a few successful cases. The question naturally arises, can this sterility be prevented by more suitable treatment of acute epididymitis. There is often a considerable amount of inflammation round the tubules of the epididymis, and I feel that if the exudate was drained away, there would be less tendency for the lumen of the vas to be permanently obliterated. 